\title{
On Signaling-Free Failure Dependent Restoration in All-Optical Mesh Networks
}

\author{
János Tapolcai*, Pin-Han $\mathrm{Ho}^{\dagger}$, Péter Babarczi*, Lajos Rónyai ${ }^{\ddagger}$ \\ * Dept. of Telecommunications and Media Informatics, Budapest University of Technology, \{tapolcai, babarczi\}@tmit.bme.hu \\ $\dagger$ Dept. of Electrical and Computer Engineering, University of Waterloo, Canada, p4ho@uwaterloo.ca \\ $\ddagger$ Computer and Automation Research Institute Hungarian Academy of Sciences and BME, ronyai@sztaki.hu
}

\begin{abstract}
Failure dependent protection (FDP) is known to achieve optimal capacity efficiency among all types of protection, at the expense of longest recovery time and more complicated signaling overhead. This particularly hinders the usage of FDP in an all-optical mesh networks. As a remedy, the paper investigates a new restoration framework that enables all-optical fault management and device configuration via state-of-the-art failure localization techniques, such that the FDP restoration process can be implemented without relying on any control plane signaling. With the proposed restoration framework, a novel spare capacity allocation problem is defined, and is further analyzed on circulant topologies for any single link failure, aiming to to gain a solid understanding of the problem. By allowing reuse of monitoring resources for restoration capacity, we are particularly interested in the monitoring resource hidden property where less or even no monitoring resources are consumed as more working traffic is in place. To deal with general topologies, we introduce a novel heuristic approach to the proposed spare capacity allocation problem, which is comprises a generic FDP survivable routing scheme followed by a novel monitoring resource allocation method. Extensive simulation is conducted to examine the proposed scheme and verify the proposed restoration framework.
\end{abstract}

\section{INTRODUCTION}

Failure dependent protection (FDP) [1]-[15] was reported as the most general approach for spare capacity allocation that can possibly achieve optimal capacity efficiency. Basically, an FDP scheme protects a working lightpath (W-LP) with multiple end-to-end protection lightpaths (P-LPs), each corresponding to a specific failure event. The pre-planned PLPs may not be disjoint from the W-LP, while the W-LP will be restored by activating one of the P-LPs according to the identified failure event that unexpectedly interrupted the W-LP. Although with the best flexibility and generality, the FDP restoration process requires a suite of real-time signaling mechanisms for failure localization, failure notification, failure correlation, and P-LP setup (or device configuration), and is considered impractical in an all-optical network where failure recovery time of a W-LP should be within a few tens of milliseconds.

To relax the stringent requirements on real-time signaling and nodal processing, various protection schemes were reported by imposing constraints on FDP to achieve respective design premises. Path protection (such as shared backup path protection SBPP) relaxes the requirement of precise failure localization. It only requires a P-LP to be disjoint from every shared risk link group (SRLG) involved in the WLP. Link/span protection [16]-[24] further restricts the traffic switchover and merging to be held at immediate upstream and downstream nodes of the failure, respectively, in order to minimize the signaling effort and recovery time. In particular, p-Cycle [19]-[24] stands for a class of link/span protection schemes where pre-configured spare capacity is allocated in a structure of a ring to restore working capacity along each span. With an ultra-fast restoration process and simple realtime management effort due to pre-configured spare capacity, p-Cycle has attracted extensive interest in the research community in the past decade, and has been widely considered in the practical operation of Internet carriers. Again, these advantages are at the expense of impaired capacity efficiency.

The merits of FDP are clear, yet the large management complexity and long recovery time hinders its acceptance to more practical purposes. To the best of our knowledge, all the existing protocols for failure notification, lightpath setup, and resource reservation have to rely on real-time control plane signaling, which becomes a fundamental barrier for any protection scheme to achieve signaling-free restoration that can be completely performed in the optical domain. Opticallayer failure localization schemes using multi-hop supervisory lightpaths (or called monitoring trails (m-trails) in the sequel) have been extensively studied [25]-[41], and are considered essential to the desired restoration process. However, none of them has taught how an FDP restoration can be incorporated for this purpose.

Motivated by the above, the paper investigates a novel framework for FDP to achieve signaling-free restoration that can be performed completely in the optical domain. The proposed framework is characterized by a number of novel designs that lead to intelligent control and autonomous fault management for ultra-fast failure restoration. Firstly, the proposed framework allows each node to localize any SRLG failure by inspecting the on-off status of the m-trails that traverse through the node. Such a node is said to be capable of achieving Local Unambiguous Failure Localization (L-UFL), and a network is called Network-wide L-UFL (NL-UFL) if all the nodes are L-UFL capable [42], [43]. Secondly, the proposed framework allows sharing of wavelength links (WLs) between P-LPs and m-trails. This is made possible since the monitoring resources are not launched with any working traffic 
and can be reused by a P-LP for working traffic restoration. Since each L-UFL node can instantly identify the failed SRLG, the required actions in response to any identified failure state can be maintained at each node in normal operation; thus all the intermediate nodes along a P-LP can start configuring their switch fabrics immediately after the identification of the failure without waiting for any failure notification and path setup request, and the switching nodes of the interrupted WLPs can switch over the working traffic to the planned P-LPs in the first place.

Based on the proposed restoration framework, we formulate a novel spare capacity allocation problem aiming to minimize the total consumption of monitoring and restoration resources by $\mathrm{m}$-trails and P-LPs, respectively. To gain deeper understanding on the problem, a polynomial time deterministic construction for optimal NL-UFL m-trail allocation is developed on circulant topologies under single link failure. Based on the construction we further analyze the upper bound on the monitoring overhead (i.e., the monitoring resources not reused by any P-LP). We prove that the monitoring resources can be completely hidden no matter how small the working traffic load is in the considered scenario. To deal with general topologies, we then introduce a heuristic approach to solve the proposed problem, where a novel $\mathrm{m}$-trail allocation scheme for multi-link SRLGs, called Network-Wide Local Link Code Construction (NL-LCC), is proposed. Extensive simulation is conducted to compare the proposed approach to p-Cycle and cooperative fast protection (CFP), which are two state-ofthe-art pre-configured protection schemes that do not rely on network layer signaling mechanisms in the restoration process.

The rest of the paper is organized as follows. Section II provides background on restoration time analysis and failure localization in all-optical mesh networks. Section III presents the proposed spare capacity allocation problem and its implementation issues. Section IV presents a novel construction on special topologies similar to real networks. Based on these results section IV presents our analytical results on the proposed problem, followed by section $\mathrm{V}$ which presents the proposed heuristic. Section VI presents the simulation results, and Section VII concludes the paper.

\section{BACKGROUND}

The section provides background of the study, specifically on restoration time analysis in the context of Generalized Multi-Protocol Label Switching (GMPLS) and review for LUFL.

\section{A. Restoration Time Analysis}

Restoration time (or recovery time) is a critical parameter of a restoration scheme. A sufficiently short restoration time is necessary for an optical layer restoration scheme not only due to the service integrity and continuity requirement, but also to make the optical layer failure events transparent to the upper layer protocols. The typical limit for restoration time is $50 \mathrm{~ms}$ inherited from SONET/SDH.
As defined in GMPLS control plane [44], a restoration process of a W-LP is composed of a number of real-time tasks after the occurrence of a failure event, until the interrupted optical flow of the W-LP is completely restored by the P-LP. Since this paper focuses on comparison of different optical layer restoration schemes, the real-time tasks considered in this study include (1) failure localization, (2) failure notification, (3) failure correlation, (4) path selection, and (5) P-LP setup. Restoration time is the time spent on the restoration process, which is modeled as:

$$
t_{R}=t_{f l}+t_{f n}+t_{f c}+t_{p s}+t_{d c}
$$

where:

$t_{f l}$ is for failure localization, which is defined as the time between the instant that the failure occurs, and that the failure is detected by some nodes close to the failure.

$t_{f n}$ is defined as the time period between the instant that some nodes that identified the occurrence of the failure send notifications to the corresponding switching node, and the the corresponding switching node receives the notifications. Note that a failure may cause multiple nodes to notify/alarm. $t_{f n}$ can be significantly reduced if the failure localization is performed at a node close to the switching node. For example, in a link/span protection scheme the failure is localized exactly at the switching node and thus $t_{f n}=0$. On the other hand, a path protection scheme usually takes much longer notification time due to multi-hop signaling via a network layer protocol.

$t_{f c}$ is defined as the time period between time instant that the switching node receives all the notifications, and the time instant that the failed SRLG is successfully identified at the switching node.

$t_{p s}$ is defined as the time for the switching node to select a right P-LP to restore the identified failure. Note that there are multiple P-LPs for a single W-LP each corresponding an SRLG failure event that may interrupt the W-LP.

$t_{d c}$ is defined as the time for setting up the P-LP, specifically between the instants that the switching node selects a right P-LP and that the P-LP is formed for traffic restoration. The typical switching time (i.e., the time to configure and set up the switching matrix of an optical cross-connect) node is $10 \mathrm{~ms}$, which is performed sequentially at every node along the P$\mathrm{LP}^{1}$, thus totally taking $40-100$ milliseconds or even longer, depending on the length and hop counts of the P-LP.

Beyond the $t_{R}$ restoration time, $t_{t s}$ is the traffic restoration completion time that is nonzero for all shared protection schemes. It is dominated by light propagation along protection routes, which is about $0.005 \cdot l_{[\mathrm{km}]}$, where $l_{[\mathrm{km}]}$ is the physical length of the protection segment. It is the time taken between the instant that the P-LP is set and the instant that the destination node receives the data flow to continue the service.

Dedicated $1+1$ protection gives a very fast restoration, where we can take $t_{f l}=t_{f n}=t_{d c}=t_{p s}=0$, and $t_{t s}$ is assumed to be a few milliseconds. Pre-configured protection

\footnotetext{
${ }^{1} \mathrm{~A}$ tell-and-go mechanism could be employed to pipeline the distributed nodal configuration process, which is nonetheless not considered in this study.
} 
(e.g., p-cycle) can achieve $25-30 \mathrm{~ms}$ restoration time by having $t_{f l}=10 \mathrm{~ms}, t_{f n}$ close to zero, $t_{d c}=10 \mathrm{~ms}$ required at the two nodes for switch fabric configuration, and a few milliseconds for $t_{t s}$.

The above two schemes generally do not rely on control signaling, but some other schemes employ a suite of multihop signaling mechanisms. In shared link/span protection, $t_{f l}$ is similar to that of p-cycle, but establishing a P-LP requires multi-hop control plane signaling, which may yield a few tens milliseconds depending on the length of the P-LP. For path protection schemes such as SBPP, the restoration time is even longer due to the multi-hop signaling and longer PLPs than that in link/span protection. A detailed evaluation on the restoration time of the traditional protection schemes can be found in [45]. At last, an FDP scheme could require the longest restoration time due to the following three reasons. (1) Since every node along the W-LP could be the switching node, $t_{f n}$ could be a multi-hop signaling process which is the longest possible. (2) Since the P-LP is not pre-configured, a regular P-LP setup process is required, which leads to the longest $t_{d c}$ compared to any other scheme. (3) Since each WLP is equipped with multiple P-LP, a non-trivial $t_{p s}$ exists and should be the longest compared to any other.

\section{B. Local Unambiguous Failure Localization (L-UFL)}

Failure localization in all-optical mesh networks using multi-hop supervisory lightpaths (S-LPs) has been extensively investigated in the past decade, and numerous approaches have been reported [25]-[33], [38]-[42]. Monitoring trails (m-trails) [40], [46] have been proposed as a monitoring structure that generalizes all the previous reported studies. An m-trail is bidirectional and could pass through a node multiple times and must pass a link twice in both directions. Therefore, an m-trail could be in any shape (e.g., simple/non-simple path, tree, and simple/non-simple cycle) whereas the transmitter and receiver can be placed at any node along the m-trail.

Fig. 1 shows an example of $\mathrm{m}$-trail solution to the network in 1(a) for localizing any single link failure, where an alarm code table (ACT) is shown in Fig. 1(b). The ACT keeps the alarm code of each link (e.g., link $(3,0)$ is assigned an alarm code 110), which further defines how the three $\mathrm{m}$-trails (i.e., $T_{1}, T_{2}$, and $T_{3}$ ) should be routed in the topology to achieve Unambiguous Failure Localization (UFL). Here, $T_{j}$ has to traverse through all the links with the $j$ th bit of the alarm code " 1 " while avoiding to take any link with the $j$ th bit of its alarm code "0". By reading the status of the three m-trails, any link failure can be unambiguously localized. For example, the darkness of $T_{1}$ and $T_{2}$ reports the failure of link $(3,0)$.

L-UFL is an advanced application of m-trail deployment. A node is said to be $L$-UFL capable if and only if the node can perform UFL by locally inspecting the on-off status of traversing m-trails. With such a definition, [43] formulated the $\mathrm{m}$-trail allocation problem via integer linear programs (ILP) when a set of nodes are required to be L-UFL capable. It was shown that the increase of the number of L-UFL capable nodes yields very mild increase of the consumed monitoring

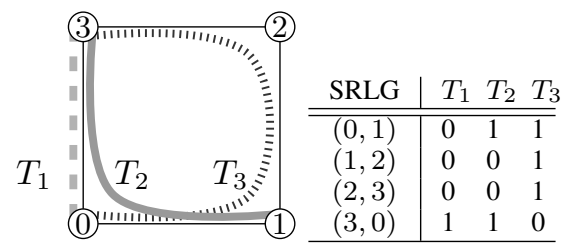

(a) Topology and m-trails(b) Alarm code table (ACT)

Fig. 1. Unambiguous failure localization (UFL) based on m-trails.

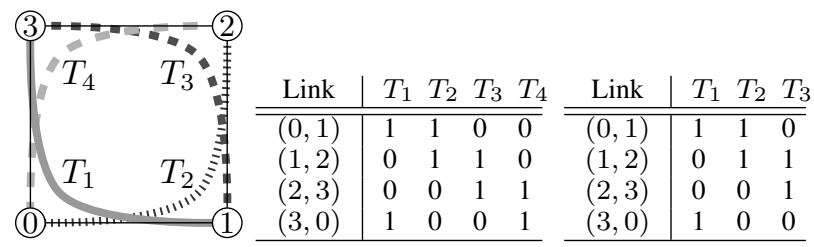

(a) M-trails

(b) Route table

(c) ACT at node 1

Fig. 2. Network-wide Local UFL (NL-UFL) via m-trails

resources. In [42] a comprehensive analysis framework is provided by having every node to be L-UFL capable, namely the Network-wide L-UFL (NL-UFL). Note that both of the above studies considered single-link SRLGs.

The problem formulation for NL-UFL m-trail allocation from [42] is reviewed as follows. The input is a network topology, which is represented by an undirected graph $G(V, E)$ with a set of links $E$ and nodes $V$, and a set of SRLGs denoted by $Z$, where each SRLG $z \in Z$ is a set of links. The task is to allocate m-trails with minimum total cover length, which is the sum of links in each m-trail in the solution and denoted by $\|T\|$, such that

(R1): Each m-trail $T_{j}$ is a connected subgraph of $G$ for $j=$ $1, \ldots, b$, where $b$ is the total number of trails.

(R2): At each node $v \in V$ each SRLG $z \in Z$ has a unique alarm code $A_{z}^{v}$, where $A_{z}^{v}=\left[a_{z, 1}^{v}, a_{z, 2}^{v}, \ldots, a_{z, b_{v}}^{v}\right]$ is a binary vector, $b_{v}$ is the total number of m-trails traversing node $v$, and the $j$ th bit of $A_{z}^{v}$, denoted by $a_{z, j}^{v}$, is $a_{z, j}^{v}=0$ if the $j$-th $\mathrm{m}$-trail traversing node $v$ is operating after failure $z$, and $a_{z, j}^{v}=1$ otherwise.

Thus for each node $v$ we define an ACT, denoted as $\underline{\underline{A}}^{v}$, which is a $|Z| \times b_{v}$ size matrix with each row as $A_{z}^{v}, \forall \bar{z} \in$ $Z$. Obviously $b \geq b_{v}$ for each $v \in V$, thus it is always a sub-matrix of the global ACT. When any failure occurs and interrupts one or a number of m-trails, node $v$ will obtain a nonzero alarm code which uniquely identifies the failed SRLG.

Note that since each m-trail is bi-directional, it is sufficient to have $T_{j}$ as a connected graph such that each traversed node can instantly obtain the on-off status of $T_{j}$. Fig. 2(a) shows an example of NL-UFL for any single-link SRLG using four m-trails in a topology with 4 nodes. Fig. 2(b) shows the routes of the four m-trails $T_{1}, T_{2}, T_{3}$, and $T_{4}$. In our case, each of the four nodes can achieve single-link UFL by inspecting the locally available on-off status of the traversing m-trails. For example, node 1 maintains an ACT as shown in Fig. 2(c), where the on-off status of $T_{1}, T_{2}$, and $T_{3}$ form an alarm code 
of three bits which uniquely maps to each possible link failure event. If node 1 finds that $T_{1}$ and $T_{2}$ become suddenly off while $T_{3}$ is still on, link $(0,1)$ is considered down and can be localized as defined in the first row of the ACT; if $T_{1}$ and $T_{2}$ are on while $T_{3}$ is off (as shown in the third row of the ACT), link $(2,3)$ is considered down. Similarly, node 0,2 , and 3 can perform UFL by maintaining their ACTs, each of which keeps the mapping between all the considered failure states and the on-off status of the traversing m-trails.

With the above, we have the amount of required monitoring resource along link $e$, denoted as:

$$
m_{e}=\sum_{1 \leq j \leq b} I_{j, e}^{T}
$$

where $I_{j, e}^{T}$ is the trail-link indicator (a.k.a global ACT) which is 1 if the $j$-th m-trail passing through link $e$, and 0 otherwise.

\section{PROPOSED FrAMEWORK}

We will first provide a detailed description on the proposed restoration framework and explain why it can achieve control signaling-free restoration in the optical domain. Then a spare capacity allocation (SCA) problem will be formulated according to the framework.

\section{A. Framework Introduction}

In a nutshell, the proposed framework integrates the FDP restoration process with state-of-the-art all-optical failure localization techniques. By equipping each node with capability of unambiguously localizing any SRLG failure (or referred to as L-UFL [42]), the switching node can precisely identify the failed SRLG by inspecting the traversing m-trails, which carries out the failure localization and failure notification tasks in the conventional restoration process without relying on any multi-hop signaling. On the other hand, since all the nodes along the P-LPs are L-UFL nodes, too, they can identify the failed SRLG using the traversing m-trails. Thus, with the prior knowledge regarding the mapping between each SRLG and switching fabric configuration, the intermediate nodes can start device configuration as soon as the failed SRLG is identified. The above restoration process demonstrates a completely signaling-free framework for FDP restoration, and its restoration time $t_{r}^{F D P}$ can be modeled as:

$$
t_{r}^{F D P}=t_{f l}^{F D P}+t_{p s}+t_{d c}^{F D P}+t_{t s},
$$

where $t_{f l}^{F D P}$ is the time by which the monitoring result converges at each node, and its upper bound is the propagation delay of the longest $\mathrm{m}$-trail; and $t_{d c}^{F D P}$ is the time for a single node to configure its switching fabric because all the intermediate nodes along the P-LP can start device configuration in parallel right after the identification of the corresponding failed SRLG; and $t_{p s}$ and $t_{t s}$ are the same as that in Eq. (1).

Clearly, the proposed restoration process can be performed completely in the optical domain because no nodal signaling required. Therefore, it demonstrates many advantages over the conventional restoration approaches where multi-hop signaling mechanisms have to be in place. The expenses paid for the advantages are the complexity in designing the $\mathrm{m}$-trails for achieving NL-UFL and corresponding resource monitoring. Besides, each node must be informed in advance regarding how it should react to any identified SRLG failure. To be specific, the switching node of any W-LP and the intermediate nodes of any P-LP have to know in which circumstances they should switch over the working traffic and configure their devices to form the P-LP. Such information can be maintained at each node and is updated whenever any W-LP is set up or torn down, or any newly established P-LP traverses through the node.

The rest of the section introduces a novel spare capacity allocation problem under the proposed framework.

\section{B. Problem Formulation}

A key issue to achieve the proposed FDP restoration framework is an approach to determine how m-trails and P-LPs under FDP are allocated, when we are provided with the network topology and a set of W-LPs. The spare capacity problem is different from any previously reported ones since it considers the allocation of both monitoring resources (for $\mathrm{m}$-trails) and restoration resources (for P-LPs). The input of the problem is as follows.

1) The network topology, which is represented by an undirected graph $G(V, E)$. For the sake of simplicity we assume infinite capacity on each link. The cost for allocating a unit of spare capacity on link $e$ (the administrative weight) is denoted as $c_{e}, \forall e \in E$, which is taken as a constant in this study.

2) The set of SRLGs, which is denoted by $Z$. Each SRLG $z \in Z$ contains one, or at most two and adjacent links.

3) A set of W-LPs denoted as $W=W_{1}, W_{2}, \ldots, W_{k}$, where $k$ is the total number of W-LPs. Each $W_{j}$ is a path in $G$ between nodes $s_{j}$ and $d_{j}$ for $j=1, \ldots, k$.

The proposed SCA problem is to minimize the total spare capacity (denoted by $u_{e}$ ) while achieving: $(i)$ a feasible solution for NL-UFL m-trail allocation under multi-link SRLGs, and ( ii) restoration capacity allocation for FDP.

An important feature of the proposed approach is that the spare capacity taken by the m-trails can be reused by any P-LP. This is a reasonable assumption since both spare capacity by $\mathrm{P}$ LPs and $\mathrm{m}$-trails are not launched with working traffic during normal operations. Once there is a failure, all monitoring resources for the m-trails can be released and reused by the P-LPs, thanks to NL-UFL which enables all the nodes to instantly react upon the identified failure. With this, we have:

$$
u_{e}=\max \left\{m_{e}, p_{e}\right\}
$$

where $m_{e}$ denotes the required monitoring resources for the m-trails as given in (2), and $p_{e}$ denotes the restoration capacity for FDP on link $e \in E$ that is formalized as follows.

1) Restoration Capacity $p_{e}$ : For each $W_{j}, 1 \leq j \leq k$, a set of P-LPs denoted as $P_{j}^{z}, \forall z \in Z_{j}$, is determined such that they are simple paths with source $s_{j}$ and destination $d_{j}$ 
while disjoint from each $z$. Let $W^{z} \subseteq W$ be a subset of WLPs, which are the W-LPs possibly interrupted by failure $z$. Let $Z_{j} \subseteq Z$ be a subset of SRLGs traversed by $W_{j}$. The amount of reserved working capacity along link $e$ is denoted as $q_{e} \forall e \in E$; formally $q_{e}=\sum_{1 \leq j \leq k} I_{j, e}^{W}$, where $I_{j, e}^{W}$ is a working path-link indicator which is 1 if $W_{j}$ passes through link $e$, and 0 otherwise. Let $I_{j, e}^{P, z}$ be a protection path-link indicator which is 1 if $P_{j}^{z}$ traverses $e$, and 0 otherwise. Let the restoration capacity along link $e$ in the failure event of $z$ be denoted by $p_{e}^{z}$. We have the following relation:

$$
p_{e}^{z}=\sum_{1 \leq j \leq k} I_{j, e}^{P, z}-\sum_{W_{j} \in W^{z}} I_{j, e}^{W}
$$

where the term $\sum_{W_{j} \in W^{z}} I_{j, e}^{W}$ stands for the free capacity gained by stub release at link $e$ after the failure event at $z$ which interrupted all $W_{j} \in W^{z}$.

The condition for $p_{e}$ to restore every affected W-LP $W_{j} \in$ $W^{z}$ by failure $z$ is:

$$
p_{e} \geq \max _{z \in Z} p_{e}^{z} .
$$

In general, the monitoring resource consumption is determined only by the topology and SRLGs considered in the problem regardless of the traffic, which serves as a constant expense; on the other hand, the amount of restoration resources heavily depends on the amount of working traffic. Thus, when the amount of working capacity increases, more monitoring resources will be reused due to the increased restoration resource consumption, and gradually all the monitoring resources are reused. We define the monitoring resources that are not shared by any P-LP as monitoring overhead, denoted by $r_{e}=\max \left\{0, m_{e}-p_{e}\right\}$. The monitoring resources are expected to be completely hidden by P-LPs, provided that we have sufficient working capacity in the network, resulting zero monitoring overhead. This is also referred to as the monitoring resource hidden property where all WLs taken by m-trails are also reserved by P-LPs.

\section{PRoblem ANALYSis}

With the SCA problem formulated in the previous section, we are particularly interested in the monitoring resource hidden property, which defines and quantifies in what circumstance the monitoring WLs are all reused by the P-LPs. In other words, there is not any additional resource consumed due to the deployment of the m-trails and thus the spare capacity consumption is the same as the conventional FDP which is optimal among all the protection schemes. As a first study into the proposed framework, we analyze the problem under single-link SRLGs in circulant graphs, aiming to gain deeper understanding on the performance behaviour of the proposed framework.

The section firstly introduces a novel construction toward essential optimal NL-UFL solutions for circulant graphs $C_{n}(1,2)$. A circulant $C_{n}(1,2)$ graph has nodes $V=$ $\{0,1, \ldots, n-1\}$ with each node $j$ adjacent to $[(j+1) \bmod n]$ and $[(j+2) \bmod n]$. An example of circulant $C_{9}(1,2)$ is given in Fig. 3. Compared to other special graphs such as

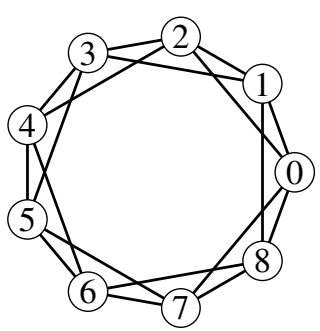

Fig. 3. An example of circulant graph $G=C_{9}(1,2)$

line, star, and complete graphs, circulant graphs are considered similar to practical carrier topologies. On the basis of the construction on circulant graphs, in the next section we investigate the monitoring resource hidden property of the problem.

\section{A. NL-UFL construction for circulant graphs}

Circulant graphs $C_{n}(1,2)$ under single-link SRLGs are considered. We define an NL-UFL m-trail solution as essentially optimal if the gap to the optimal total monitoring resource consumption is less than $O(\log |E|)$ in every case.

Theorem 1: Circulant graph $G=C_{n}(1,2)$ can be covered with $b=\left\lceil\log _{2}(2 n+1)\right\rceil \mathrm{m}$-trails for NL-UFL, where each m-trail is a spanning sub-graph of $G$, and the total cost is $b n+1$.

Proof: To prove the theorem, our approach is via a novel construction that generates a set of connected subgraphs of $G$ as m-trails, which can be proved to achieve NL-UFL for any single-link failure.

1) The proposed construction: Let an alarm code of each link in $G$ be $b$ bits in length. The code $[11 \ldots 1]^{2}$ is assigned to edge $(0,1)$, while the other edges $(v, v+1)$ are each assigned with an alarm code that is the binary representation of the value $v+1$. Note that, the first bit of these codes is always 0 and the rest is a nonzero bit vector. Edge $(v, v+2)$ is assigned with a code which is bitwise complement of the alarm code of $(v, v+1)$ where $v \neq 0$. Thus, the first bit of these codes for $(v, v+2)$ is 1 . Besides, the complementary pair of codes $[00 \ldots 01]$ and $[11 \ldots 10]$ are not assigned to any edge. Finally, edge $(0,2)$ is associated with the bit vector $[00 \ldots 01]$.

The set of m-trails is deployed in such a way that $T_{j}$ traverses through all the edges with their $j$ th bit position as 1 while disjoint from any edge with the $j$ th bit position as 0 .

2) Correctness of the constructed m-trail solution: Since we have:

$$
2^{b}-2 \geq 2 n-1,
$$

it is clear that such an assignment generates a unique alarm code for each link. In the rest of the proof we show that for any $1 \leq j \leq b$, the subgraph $T_{j}$ corresponding to links with their $j$ th bit position of alarm codes as 1 is weakly connected and spans the whole vertex set. To make the proof easily presented, let us take each edge in $G$ as directed counter-clockwise, i.e., edge $(v, v+1)$ is directed from $v$ to $v+1$, and similarly $(v, v+2)$ from $v$ to $v+2$. It is sufficient to show that every

\footnotetext{
${ }^{2} x \ldots x$ denotes a code fragment with $x$ in every bit position
} 
directed cycle from the edges of $T_{j}$ passes through node 0 . This is due to the following two facts: (1) the codes of $(v, v+1)$ and $(v, v+2)$ as $v \neq 0$ are bitwise complement to each other, thus $T_{j}$ must connect from $v$ to either $v+1$ or $v+2$, this results every cycle to traverse the circulant graph counter-clockwise through either node 0 or 1 ; and (2) when $v=0$, edge $(0,1)$ is traversed by any directed cycle since it has a code $[11 \ldots 1]$. The above two facts make the outdegree in $T_{j}$ of every node of $G$ at least $1^{3}$.

3) Number of m-trails for NL-UFL: To evaluate the total cost, the alarm codes for the directed edges leaving vertex 0 contain exactly $b+1$ values of 1 (i.e., edge $(0,1)$ has $b+1$ $\mathrm{m}$-trails to traverse through). For the other edges $(v, v+1)$ and $(v, v+2)$ with $v \neq 0$, each of them has $b$ values of 1 . This implies that the total number of $1 \mathrm{~s}$ is $b n+1$ as claimed.

The proposed construction is optimal in terms of the number of $\mathrm{m}$-trails as shown by the information theoretic lower bound $b \geq\left\lceil\log _{2}(|E|+1)\right\rceil$ and $|E|=2 n$. The solution is a feasible NL-UFL solution because each m-trail spans the whole network following the unique alarm code of each link. Next we show that the proposed construction yields essentially optimal NL-UFL solutions in terms of the total cost.

\section{B. Lower bound on total cover length}

Let $G$ be a connected graph with $n$ nodes and $m$ edges, and $T_{1}, \ldots T_{b}$ give an NL-UFL solution. The total cover length of an NL-UFL solution, denoted by $\|T\|$, is defined as the number of links (or WLs) consumed by all the m-trails, which is the total hop count traversed by the m-trails.

Let $r(T)$ denote the number of nodes $\mathrm{m}$-trail $T$ has. These nodes are aware of the on-off status of $T$. A trivial upper bound on $r(T)$ is $|T|+1$ where $|T|$ is the number of edges traversed by $T$; or formally

$$
r(T) \leq|T|+1 .
$$

Theorem 2: The total cover length for an NL-UFL solution is at least

$$
\|T\| \geq \begin{cases}\frac{n \cdot m}{m+2} \log _{2}(m), & n-1<2 m,(4 a) \\ m+(n-1) \log _{2}\left(\frac{n-1}{2}\right) & \text { otherwise. }\end{cases}
$$
follows

Proof: Let $\omega\left(\left|T_{i}\right|\right)$ be a cost function for m-trail $T_{i}$ as

$$
\omega\left(\left|T_{i}\right|\right)= \begin{cases}\frac{2\left|T_{i}\right|}{1+\left|T_{i}\right|} & \text { if }\left|T_{i}\right| \leq n-1, \\ \frac{2\left|T_{i}\right|}{n} & \text { otherwise. }\end{cases}
$$

Let us define a matrix $\Omega$ with $n$ columns and $b$ rows, where

$$
\omega_{v, i}=\left\{\begin{aligned}
\frac{\left|T_{i}^{v}\right|}{r\left(T_{i}^{v}\right)} & \text { the } i \text { th m-trail traverses node } v, \\
0 & \text { otherwise. }
\end{aligned}\right.
$$

\footnotetext{
${ }^{3}$ To be more specific, the outdegree of $v$ is exactly 1 , if $v \neq 0$, and the outdegree of vertex 0 is larger or equal to 1 .
}

The size of $T_{i}$ can be expressed as

$$
\sum_{v=1}^{n} \omega_{v, i}=\sum_{v \in T_{i}} \frac{\left|T_{i}\right|}{r\left(T_{i}\right)}=\left|T_{i}\right|
$$

Thus we have

$$
\sum_{i=1}^{b} \sum_{v=1}^{n} \omega_{v, i}=\sum_{i=1}^{b}\left|t_{i}\right|=\|T\|
$$

which can be reordered as

$$
\begin{aligned}
& \|T\|=\sum_{i=1}^{b} \sum_{v=1}^{n} \omega_{v, i}=\sum_{v=1}^{n}\left(\sum_{i=1}^{b} \omega_{v, i}\right)= \\
& =\sum_{v=1}^{n}\left(\sum_{i \mid v \in T_{i}} \omega_{v, i}\right) \geq \sum_{v=1}^{n}\left(\sum_{i \mid v \in T_{i}} \frac{\omega\left(\left|T_{i}\right|\right)}{2}\right) \geq \frac{n \Omega}{2}
\end{aligned}
$$

where $\Omega$ is a lower bound on $\sum_{i}^{b} \omega\left(\left|T_{i}\right|\right)$. The first inequality is a consequence of 3 . Note that the function $\omega(t)$ satisfies the conditions in the Definition 1 in [47], because $\omega(t+1) \geq \omega(t)$, $\omega(1)=1$, and $\frac{\omega(t+1)}{t+1} \leq \frac{\omega(t)}{t}$. Although the problem scenario with $\mathrm{m}$-trails is slightly different than finding a separating system as in the gnCGT problem since none of the items can have all zero code. However, such a constraint further restricts the problem, thus the lower bounds derived in the gnCGT problem remains valid.

Consider the case (4a). Then we have $t=\left|T_{i}\right| \leq \frac{m}{2} \leq n-1$, hence the cost function here is (5b). By Theorem 1 of [47] we have

$$
\Omega \geq \min _{1 \leq t \leq \frac{m}{2}} \frac{2 t}{1+t}\left(\log _{2} t+\frac{m}{t}-1\right)
$$

where inside the min there is an decreasing function of $t$ as proved in Lemma 3 in [47]. Thus, it leads to

$$
\begin{aligned}
& \Omega \geq \frac{2 \frac{m}{2}}{\frac{m}{2}+1}\left(\log _{2}\left(\frac{m}{2}\right)+\frac{m}{\frac{m}{2}}-1\right)= \\
& =\frac{2 m}{m+2}\left(\log _{2}(m)-1+2-1\right)=\frac{2 m}{m+2} \log _{2}(m) .
\end{aligned}
$$

Putting it together with (9) we get (4a).

We prove (4b) by applying Theorem 1 from [47]. We obtain

$$
\begin{aligned}
\Omega \geq \min _{1 \leq t \leq \frac{m}{2}} \omega(t)\left(\log _{2} t+\frac{m}{t}-1\right)= \\
\min \left\{\min _{1 \leq t \leq n-1} \frac{2 t}{1+t}\left(\log _{2} t+\frac{m}{t}-1\right),\right. \\
\left.\min _{n-1 \leq t \leq \frac{m}{2}} \frac{2 t}{n}\left(\log _{2} t+\frac{m}{t}-1\right)\right\}
\end{aligned}
$$

where inside the first min there is a decreasing function of $t$ (Lemma 2 in Appendix), while inside the second min there is an increasing function of $t$ (Lemma 3 in Appendix). The minimum is attained at $t=n-1$. It leads to

$$
\begin{aligned}
\Omega \geq \frac{2(n-1)}{n}\left(\log _{2}(n-1)+\frac{m}{n-1}-1\right) \\
=\frac{2}{n}\left((n-1) \log _{2}\left(\frac{n-1}{2}\right)+m\right)
\end{aligned}
$$


Putting it together with (9) we get (4b).

Corollary 1: The m-trail construction in Theorem 1 is an essentially optimal NL-UFL solution.

Proof: The average nodal degree is 4 , thus $m=2 n$.The total monitoring capacity of the construction of Theorem 1 is $n\left\lceil\log _{2}(2 n+1)\right\rceil$. According to Theorem 2 the total cost is at least

$$
\begin{aligned}
& m+(n-1) \log _{2}\left(\frac{n-1}{2}\right)= \\
& 2(n-1)+2+(n-1)\left(\log _{2}(2 n-2)-2\right)= \\
& 2+(n-1)\left(\log _{2}(2 n-2)\right)
\end{aligned}
$$

Clearly, the gap is at most $2 \log _{2}(|E|)$.

\section{Lower Bound on the Spare Capacity}

Lemma 1: Let $K$ be a set of links that form a cut in $G$. The spare capacity along the links in $K$ is at least

$$
\frac{1}{|K|-1} \sum_{e \in K} q_{e} \leq \sum_{e \in K} p_{e} .
$$

Proof: In case link $e$ fails, protection routes must be able to circumvent $e$ via the the other links in $K$, thus the spare capacity along those links is at least $q_{e}$, formally

$$
q_{e} \leq \sum_{f \in K, e \neq f} p_{f}
$$

There are $|K|$ such inequalities for $1 \leq e \leq|K|$. By summing up these inequalities we get

$$
\sum_{e \in K} q_{e} \leq \sum_{e \in K} \sum_{f \in K, e \neq f} p_{f}=(|K|-1) \cdot \sum_{e \in K} p_{e} .
$$

Finally, dividing both sides by $(|K|-1)$ we get Eq. (15).

Corollary 2: Let $K^{1}, \ldots, K^{k}$ be a pairwise disjoint cuts. Then

$$
\mathcal{M} \geq \sum_{1 \leq l \leq k} \sum_{e \in K^{l}} \frac{c_{e} q_{e}}{\left|K^{l}\right|-1} .
$$

Corollary 3: Let $N_{G}(v)$ denote the set of links adjacent to node $v$ and $\Delta_{v}=\left|N_{G}(v)\right|$

$$
\mathcal{M} \geq \sum_{v \in V} \sum_{e \in N_{G}(v)} \frac{c_{e} q_{e}}{\Delta_{v}-1} .
$$

As a rule of thumb, the minimal ratio of spare to working capacity can be estimated by $1 /(\bar{\Delta}-1)[1]$, where $\bar{\Delta}=2|E| /|V|$ is the average nodal degree. It was proved for the case when the working capacity is the same on every link [1]. This can be also deduced from Corollary 3 by applying the inequality of arithmetic and harmonic means.

\section{Dominance of Monitoring Resources}

With the proposed NL-UFL construction in the $C_{n}(1,2)$ topologies, we are interested in whether and how much monitoring resources can be hidden by restoration resources, and in what condition it will happen. Let the average working capacity per link, the average restoration capacity per link, and average monitoring capacity per link, be denoted by

$$
\bar{q}=\frac{1}{|E|} \sum_{e \in E} q_{i}, \quad \bar{p}=\frac{1}{|E|} \sum_{e \in E} p_{i}, \quad \bar{m}=\frac{1}{|E|} \sum_{e \in E} m_{i},
$$

respectively. Formally, the dominance of monitoring resources occurs when $\bar{m} \geq \bar{p}$, which serves as a sufficient condition that additional monitoring resources are required by FDP on top of the restoration capacity .

Let $\theta$ measure the traffic demand as a percentage of $s-d$ pairs that are loaded with a W-LP. For example, $\theta=100 \%$ means each $s-d$ pair (i.e., $|V|(|V|-1) / 2$ ) is connected by a W-LP. It is clear that with smaller $\theta$, the dominance of monitoring resources is more likely to happen. There naturally comes up an interesting question: with a specific topology, for which values of $\theta$ will make the monitoring resources dominant? Is there a lower bound on $\theta$, say $1 \%$, below which $\bar{m}<\bar{p}$ is unconditionally true? In the following theorem we show that there is no such a lower bound on $\theta$ in a circulant topology $G=C_{n}(1,2)$ under single-link SRLGs.

Theorem 3: For any positive $\theta>0$, there exists a topology where the monitoring resources will never dominate the spare capacity (i.e., $\bar{m}<\bar{p}$ ).

Proof: We pick the circulant graphs $G=C_{n}(1,2)$ with unit cost along each link for the proof of the theorem. Let $G=$ $C_{n}(1,2)$ contain a set of nodes denoted as $0,1, \ldots, n-1$, and edges denoted as $(v, v+1)$ and $(v, v+2)$, for $v=0, \ldots, n-1$, where the addition is understood modulo $n$. Let us call the edges $(v, v+1)$ by on-cycle edges and the rest chordal edges (see also Fig. 3). Let $G$ be launched with a set of shortestpath routed W-LPs denoted as $W=W_{1}, \ldots, W_{k}$, such that $k \leq \theta \cdot \frac{n(n-1)}{2}$. Let $h=\max \left\{5,\left\lceil\frac{1}{\theta}\right\rceil\right\}$ and $n=4 h \geq 20$. Nodes $s$ and $d$ are connected with a W-LP along the shortest path route if $s-d \equiv 0 \bmod h$. In this case the number of directed connections is $k=\frac{3}{2} n$, because each source node $s$ is connected with $s+h, s+2 h$ and $s+3 h$, where addition is understood modulo $n$. Therefore, the total number of directed connections is

$\theta \cdot \frac{n(n-1)}{2}=\theta \cdot 4 \cdot \max \left\{5,\left\lceil\frac{1}{\theta}\right\rceil\right\} \frac{n-1}{2} \geq 2(n-1) \geq \frac{3}{2} n=k$

Next, we define a set of disjoint cuts $K^{1}, \ldots, K^{n / 4}$, where $K^{i}$ contains 6 links $(v-1, v+1),(v, v+1),(v, v+2)$ for both $v=2 i$ and $v=2 i+n / 2$. Each cut $K^{i}, i=1, \ldots n / 4$ separates the graph into two equal-size fragments with $\frac{n}{2}$ nodes, thus the number of W-LPs passing through the cut is at least $n$, because for each source node $s$ the number of possible destination nodes in the other side of the cut is 2, thus there are at least $n$ for which $s-d \equiv 0 \bmod h$ holds.

According to Corollary 2 we have $\mathcal{M} \geq \frac{1}{5} \cdot n \cdot \frac{6}{4} n$, where $\frac{6}{4} n$ is the total number of links in the cuts $K^{1}, \ldots, K^{n / 4}$. Note that, the number of links $|E|=2 n$, thus $\bar{p}=\frac{\mathcal{M}}{2 n}$. According to Theorem 1, the total monitoring capacity of the bm-trails is $n\left\lceil\log _{2} n+1\right\rceil+n$. Thus we have $\bar{m}=\frac{1}{2}\left(\left\lceil\log _{2} n+1\right\rceil+1\right)$. Therefore, $\bar{m}<\bar{p}$ holds when $\left\lceil\log _{2} n+1\right\rceil+1<\frac{3}{10} \cdot n$ which is always true for $n \geq 20$. 
It is important to note that although sufficient, the dominance of monitoring resources is not necessary for nonzero monitoring overhead. To be specific, such dominance becomes a necessary condition for nonzero monitoring overhead only in extreme situations like in ring networks with a minimum cut of two links.

\section{General Topologies with Multi-Link SRLGs}

We approach the proposed SCA problem by firstly solving the m-trail allocation problem for multi-link SRLGs, followed by the solution of FDP restoration capacity allocation.

\section{A. NL-UFL for Multi-Link SRLGs}

We propose a novel $\mathrm{m}$-trail allocation scheme for NL-UFL under multi-link SRLGs, called NL Link Code Construction (NL-LCC). The basic idea of NL-LCC is to successively and incrementally construct the alarm code table (ACT) at each node. A similar idea was explored in our previous work [33]; however, different from any previously reported scheme, NLLCC meets all the desired features of the proposed framework which incorporates in-band information, deals with multi-link SRLGs, and can achieve NL-UFL.

Let $\underline{A}^{v}$ at node $v$ denote the ACT of $v$, which is a binary matrix on all the m-trails traversing through $v$, with each entry $a_{z, j}^{v}=1$ if $T_{j}$ passes SRLG $z, a_{z, j}^{v}=0$ if $T_{j}$ is disjoint from $z$, and "x" (i.e., a don't care bit) if it does not affect the NLUFL property by having $T_{j}$ pass through $z$ or not. The pseudo code of NL-LCC is given in Algorithm 1 and is explained step by step as follows.

In Step (3), each node has an initial ACT generated based on the terminated W-LPs, where $a_{z, j}^{v}=1$ if $W_{j}$ terminated at node $v$ passes through $z$, and 0 otherwise. Then, the heuristic enters the loop in Step (4) - (5) which checks each pair of SRLGs to ensure their alarm codes are different and distinguishable at any node $v$. The heuristic targets at that all the SRLG alarm codes are unique by flipping as few don't care bits to 0 or 1 as possible.

If two SRLG $z_{1}$ and $z_{2}$ are found with a common alarm code (i.e., $A_{z_{1}}^{v}=A_{z_{2}}^{v}$ in Step (6)), the heuristic first checks if the last bit of each alarm code is $x$ (Step (7)). If not, in Step (8) one more bit is appended and tapped with a don't care bit $x$, where the length of alarm codes at $v$ is increased by one (i.e., $b_{v}=b_{v}+1$ ).

Note that ensuring the last bit position of SRLG alarm code as $x$ is necessary for the guaranteed success in distinguishing the alarm code of SRLG $z_{1}$ and $z_{2}$ through Steps (9)-(18).

In Step (10) we iterate through each link $e \in$ $\left(z_{1} \cup z_{2}\right) \backslash\left(z_{1} \cap z_{2}\right)$ from the symmetric difference of $z_{1}$ and $z_{2}$ (e.g., $e \in z_{1}, e \notin z_{2}$ ) and in Step (11) we iterate through every bit position $j$. In Step (12) if the $j$ th bit of the alarm code of $e$ is $x$ or 1 , and $\forall f \in z_{2}$ the $j$ th position is $x$ or 0 , we treat link $e$ and position $j$ as a good candidate for distinguishing the failure of SRLG $z_{1}$ and $z_{2}$. Next, in Step (13) we evaluate the cost of this candidate, which is the number of don't care bits among $a_{e, j}^{v}$, and $a_{f, j}^{v}, \forall f \in z_{2}$. Our goal is to select the possible link $e$ and position $j$ where the fewest number of bits should be set to 0 or 1 , and thus most don't care bits remain for the next iterations, which is stored in the working variables $e_{m}$ and $j_{m}$ in Step (15). Note that, such a bit position always exists because the last bit is $x$ due to Step (8). Next, the best candidate is selected in Step (16), and bit $j_{m}$ of link $e_{m}$ is set 1 , while $\forall f \in z_{2}$ is set 0 at position $j_{m}$. In such a way we can ensure $a_{z_{1}, j_{m}}^{v} \neq a_{z_{2}, j_{m}}^{v}$, which will remain unchanged regardless of the future iterations.

Finally, we need to make sure that all the information is local at node $v$; thus we search for the shortest path in $G$ between node $v$ and the closest terminal node of $e_{m}$ through links $f$ with $a_{f, j_{m}}^{v}=\{1, x\}$ in Step (17), and set those bits to 1 in Step (18).

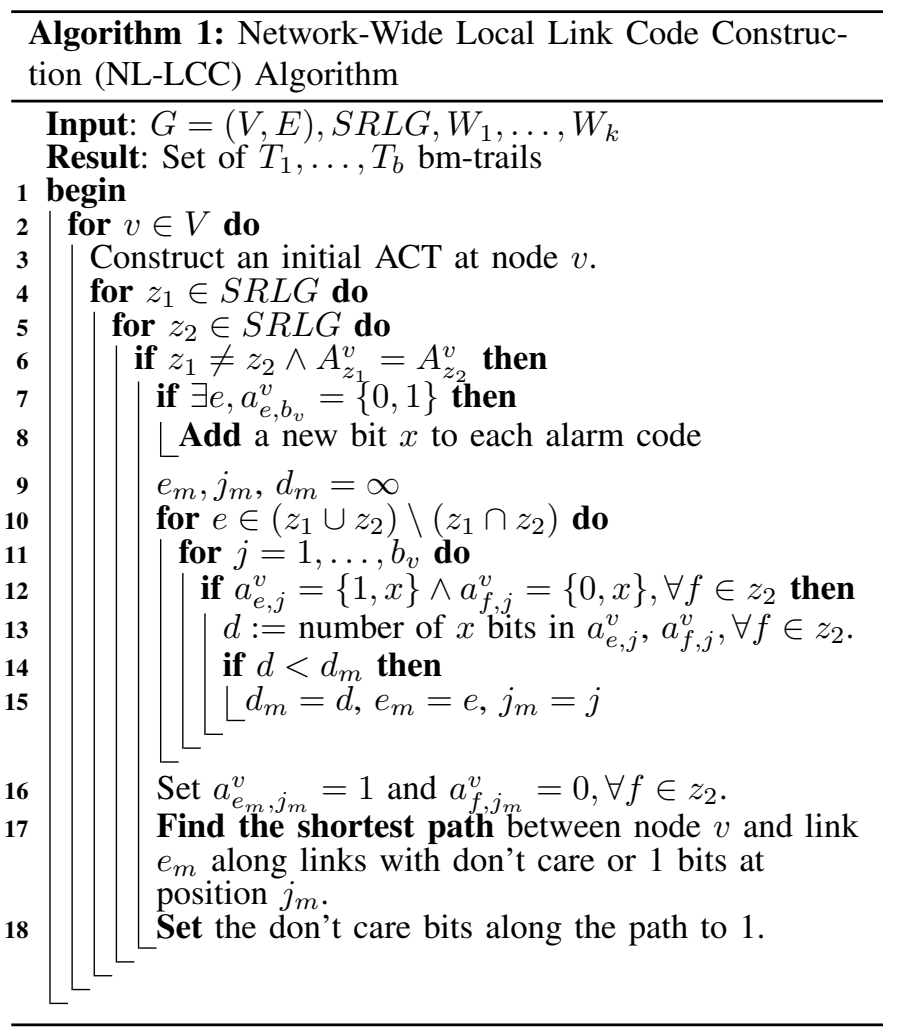

\section{B. FDP Restoration Capacity Allocation}

The restoration capacity allocation problem for FDP has been extensively investigated in the past decades, which is formally called Spare Capacity Placement/Allocation for Path Restoration with stub release. Detailed descriptions can be found in [1], [2], [4], [48, Chapter 6], [49, Chapter 9.5.2] for both ILP formulations and heuristic approaches. Since we have positioned our study on a signaling-free FDP-based restoration process via all-optical failure localization, we do not focus on novel FDP restoration capacity allocation schemes; instead, we would adopt state-of-the-art FDP spare capacity allocation schemes for the proof of concept and facilitation of performance analysis. To be specific, the ILP in [49] and Successive Survivable Routing (SSR) algorithm in [4] are implemented in Section VI for comparison with other counterparts as well 
as for understanding the performance behaviors under the monitoring resource hidden property, respectively.

\section{Vi. Performance Evaluation}

\section{A. Comparison of Signaling-free Protection Methods}

We first compare the proposed approach with a couple of reported pre-configured protection schemes, namely p-Cycle and Cooperative Fast Protection (CFP) [20]. We implemented an ILP for each of the three schemes, specifically the ones in [23] for p-Cycle, [20] for CFP, and [49, Chapter 9.5.2] for FDP that is required in the proposed approach. Note that the ILP for p-Cycle in [23] is enumeration-free and considered as a general, yet efficient ILP formulation that can achieve the optimal capacity efficiency. We employed the same link cost values as [20], [23], and [24], respectively, to ensure that the experiment environments are completely in line with the previous art, in which W-LPs with $\lambda$ WLs were shortest-path routed across the $s-d$ pairs in each case. The results were obtained by solving the ILPs using CPLEX v.11, all with a zero gap to the optimal.

1) Under Single-Link SRLGs: The first two columns of Table I show the results on single-link SRLGs using Smallnet [20] topology (10 nodes, 22 edges) and COST239 [23] network (11 nodes, 26 edges). The proposed approach consumed a total cost of $\max \{48,11 \lambda\}$ in Smallnet, compared with $17 \lambda$ and $13 \lambda$ by p-Cycle and CFP respectively; and a total cost of $\max \{35665,22660 \lambda\}$ in COST239, compared with $32760 \lambda$ by p-Cycle. Therefore, when $\lambda \geq 4$ in Smallnet and $\lambda \geq 2$ in COST239, the proposed signaling-free restoration framework will outperform p-Cycle and CFP; and with larger $\lambda$ (i.e., with more working capacity), the advantage of the proposed approach gains more advantages. Note that by assuming constant link cost and unlimited link capacity, a number of $\lambda$ W-LPs are loaded along a common route for each $s-d$ pair, which is expected to consume $\lambda$ times more restoration resources compared to single W-LP.

2) Under Multi-Link SRLGs: The last column of Table I shows the results with all single-link and dual-link SRLGs on NSF network (14 nodes, 22 links). We have adopted the problem instance in [24] for comparison, which is, to the best of our knowledge, the only previously reported study that provides an ILP for static multi-link SRLG p-Cycle design. Note that all the other studies for multi-link SRLGs using p-Cycles have focused on reconfiguration, rerouting, and dynamic reconfiguration of spare capacity, which do not fit into the targeted scenario. As shown in Table I, p-Cycle takes several times higher cost than the proposed approach even when $\lambda=1$ (i.e., 965 versus 302). This clearly shows that the proposed restoration framework gains even more advantage when multi-link SRLGs are considered.

\section{B. Monitoring Resources Hidden}

We have seen that the proposed approach is outperformed by its counterparts when the working traffic load is small, but will become much more efficient when the number of

\begin{tabular}{|l||c|c||c|c||c|c|}
\hline \multicolumn{1}{|c||}{} & \multicolumn{2}{c|}{$\begin{array}{c}\text { Smallnet [20] } \\
\text { single failures }\end{array}$} & \multicolumn{2}{c|}{$\begin{array}{c}\text { COST239 [23] } \\
\text { single failures }\end{array}$} & \multicolumn{2}{c|}{$\begin{array}{c}\text { NSF [24] } \\
\text { dual failures }\end{array}$} \\
\hline & $\mathcal{M}$ & $t[s]$ & $\mathcal{M}$ & $t[s]$ & $\mathcal{M}$ & $t[s]$ \\
\hline \hline P-cycle & $17 \lambda$ & 17.22 & $32760 \lambda$ & 34.68 & $965 \lambda$ & n/a \\
\hline CFP & $13 \lambda$ & 39004 & n/a & n/a & n/a & n/a \\
\hline \hline FDP & $11 \lambda$ & 0.41 & $22660 \lambda$ & 8.36 & $134 \lambda$ & 1485 \\
\hline M-trail & 48 & 3.53 & 35665 & 7.12 & 302 & 1319 \\
\hline
\end{tabular}

TABLE I

THE SPARE BANDWIDTH $(\mathcal{M})$ AND RUNNING TIME $(t[s])$ OF THE PROPOSED RESTORATION FRAMEWORK COMPARED WITH P-CYCLES AND CFP (W-LPS WITH $\lambda$ BU).

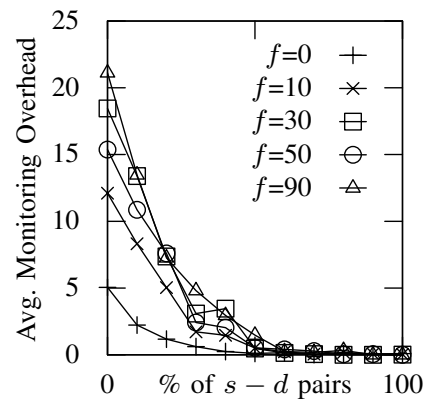

(a) COST266 network

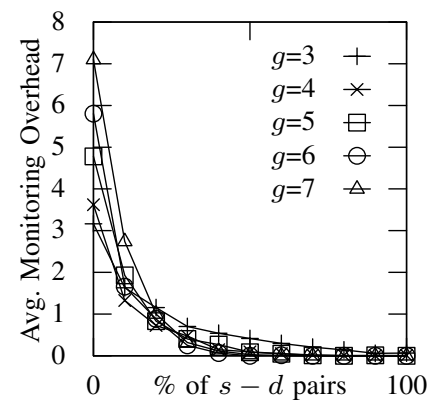

(b) Random networks with $(f=0)$
Fig. 4. Average monitoring overhead.

W-LPs increases, and the monitoring resource can be completely hidden by the restoration capacity for FDP. Therefore, we claim that the proposed approach can achieve the same capacity efficiency as conventional FDP provided the network is loaded with sufficiently large working capacity. This subsection provides extensive simulation results in a wide range of network topologies and SRLG densities, so as to gain deeper understanding on the monitoring resource hidden property under the proposed restoration framework.

The randomly generated planar graphs are classified according to parameter $g$, which is the length of the longest inner face contained in the graph. Clearly, graphs with smaller values of $g$ are considered more densely meshed; and in the generated graphs, the average nodal degree of each graph ranges from 5.4 (for $g=3$ ) to 2.76 (for $g=7$ ). We adopted SRLGs with single link and dual adjacent links, and the SRLG density considered in the problem is parameterized by a dual failure density parameter, denoted by $f$, which indicates the fraction $f$ of all dual adjacent link SRLGs under consideration.

We evaluate the resource consumption in the proposed framework by increasing the working traffic, which is defined as the percentage of $s-d$ pairs that are interconnected by a W-LP (i.e., $\theta$ ). Let every W-LP and m-trail take a single wavelength of bandwidth (i.e., a single WL). The ILP in [49] was no longer used here due to the huge computation complexity; instead, we adopted SSR [4] that sequentially allocates P-LPs for each W-LP.

Fig. 4(a) and (b) show the average monitoring overhead, 

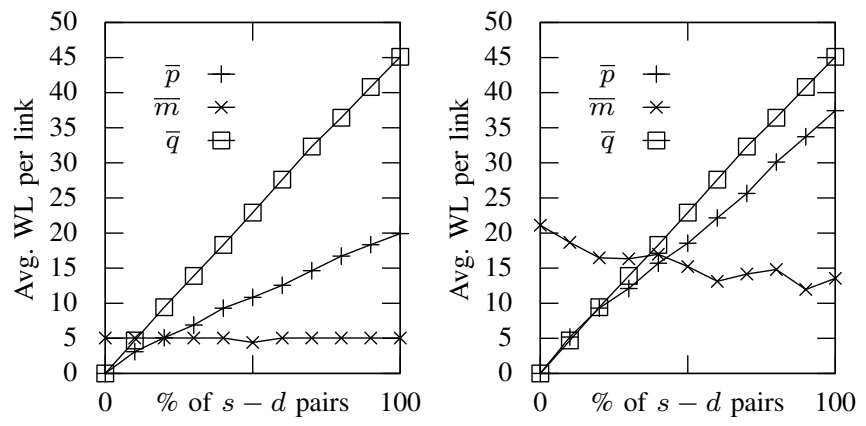

(a) Single-link SRLGs $(f=0 \%)$. (b) Adjacent dual-link SRLGs $(f=$ $90 \%)$.

Fig. 5. Average used capacity on the links in the COST266 reference network, where working, spare and m-trail capacity is denoted by $\bar{q}, \bar{p}$ and $\bar{m}$, respectively.

i.e.,

$$
\frac{1}{|E|} \cdot \sum_{e \in E} \max \left\{0, m_{e}-p_{e}\right\}
$$

as $\theta$ is increased from $0 \%$ to $100 \%$. It meets our expectation that, as the percentage of loaded $s-d$ pairs (i.e., $\theta$ ) is decreased, and/or as the percentage of dual-link adjacent SRLGs (i.e., $f$ ) increases, the monitoring overhead increases accordingly. It is interesting to observe that the topology density does not affect the monitoring overhead as shown in Fig. 4(b), because by taking a sparser topology increases the total monitoring capacity for $\mathrm{m}$-trails, meanwhile increasing the required restoration capacity for FDP, too. As a rule of thumb, we claim that the monitoring overhead is negligible if at least $50 \%$ of the node-pairs in a network are loaded with a W-LP. Further, with more than $20 \%$ of loaded $s-d$ pairs, the monitoring overhead is $1 \mathrm{WL}$ per link under single-link SRLGs, regardless the network density.

In Fig. 5(a) and (b), the average numbers of WLs per link for W-LPs, P-LPs, and m-trails are evaluated in the COST266 European reference network [42] (37 nodes, 57 links) with $f=0$ and $f=90 \%$ in (a) and (b), respectively. The intersections of the curves for average restoration capacity per link (i.e., $\bar{p}$ ) and average monitoring capacity per link (i.e., $\bar{m})$ are at $\theta=20 \%$ and $\theta=40 \%$ with an SRLG density $f=0 \%$ and $f=90 \%$, respectively. The curve of $\bar{m}$ shows the contribution adopting in-band information in the reduction of monitoring resource consumption, which is not obvious in the single-link SRLG scenario in Fig. 5(a). Nonetheless, the effect of taking in-band information becomes non-trivial when multi-link SRLGs are considered, where $\bar{m}$ decreases from 21.1 to 13.5 as $\theta$ increases from $0 \%$ to $100 \%$, as shown in Fig. 5(b). Further, the reduction of $\bar{m}$ occurs mostly in the light traffic region where the monitoring resources are more likely to be dominant.

Fig. 6(a) and (b) show the relation between the maximum total capacity required along each link (i.e., $\bar{q}+\max \{\bar{m}, \bar{p}\}$ ) and $\theta$ by using COST266 European reference network (37 nodes, 57 links), under various SRLG densities and topology

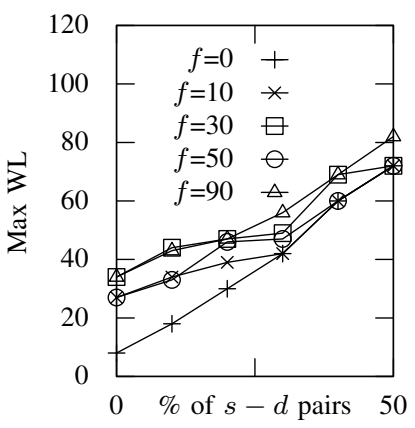

(a) COST266 network

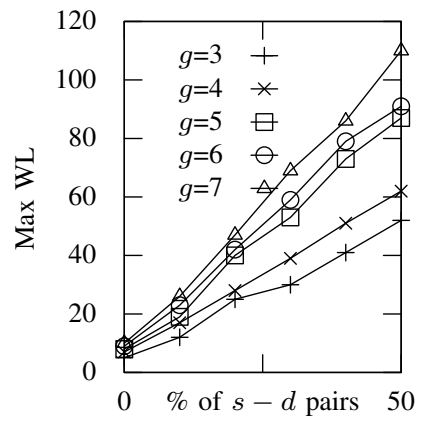

(b) Random networks with $(f=0)$
Fig. 6. Maximum overall capacity in WLs.

densities, respectively. In Fig. 6(a) when $\theta=50 \%$, the required maximum WLs is roughly 80 . This provides us a design guideline that with $80 \mathrm{WLs}$ of total capacity consumed along each link in COST266, the proposed framework can most likely achieve optimal performance by using the proposed approach, due to the fact that $\theta=50 \%$ is found to be the turning point for reaching zero monitoring overhead under multi-link SRLGs, as shown in Fig. 4(a). Further, as shown in Fig. 6(b), 30-45 WLs of overall capacity along each link is the threshold for zero monitoring overhead for single-link failures, because $\theta=20 \%$ is the turning point of zero monitoring overhead under single-link SRLGs, as shown in Fig. 4(b).

\section{CONCLUSIONS}

This paper introduced a novel restoration framework for failure dependent protection (FDP), aiming to achieve the following three desired features of optical layer protection in all-optical mesh networks: (1) a signaling-free and completely all-optical restoration process; (2) 100\% restorability for any multi-link SRLG failure event; and (3) optimal capacity efficiency as FDP. By incorporating state-of-the-art all-optical failure localization techniques, the paper first defined a novel spare capacity allocation problem that is composed of two tasks: monitoring resource allocation for m-trails, as well as restoration resource allocation for implementing FDP. The paper demonstrated how the proposed restoration process is signaling-free and all-optical with an ultra-fast and guaranteed restoration speed. Then we analyzed the proposed problem regarding its monitoring resource hidden property, where a polynomial time deterministic construction on circulant topologies was developed and proved that the dominance of monitoring resources does not occur even when traffic load (i.e., $\theta$ ) is close to zero.

A heuristic approach was developed, which includes a novel m-trail allocation scheme for NL-UFL under multi-link SRLGs, namely NL-LCC. Extensive simulation was conducted to examine the proposed approach regarding its capacity efficiency compared with p-Cycle and cooperative fast protection (CFP), as well as its monitoring resource hidden property. Our findings in the simulations are summarized as follows: (1) the proposed FDP restoration framework outperforms the two 
schemes in terms of capacity efficiency when working traffic is over some threshold, and such advantage is getting more significant when there is more working traffic; (2) a turning point exists in terms of the percentage of loaded $s-d$ pairs (i.e., $\theta$ ) which results in zero monitoring overhead, which is $\theta=50 \%$ and $\theta=20 \%$ for multi-link SRLGs and singlelink SRLGs, respectively; (3) a rule of thumb was identified to estimate whether the proposed approach achieves zero monitoring overhead using the amount of maximum occupied capacity along each link.

\section{REFERENCES}

[1] R. R. Iraschko, M. MacGregor, and W. D. Grover, "Optimal capacity placement for path restoration in STM or ATM mesh survivable networks," IEEE/ACM Trans. Networking, pp. 325-336, June 1998.

[2] Y. Xiong and L. Mason, "Restoration strategies and spare capacity requirements in self-healing atm networks," IEEE/ACM Trans. Networking, vol. 7, no. 1, pp. 98-110, Oct. 1999.

[3] H. Choi, S. Subramaniam, and H. Choi, "Loopback recovery from double-link failures in optical mesh networks," IEEE/ACM Trans. Networking, vol. 12, no. 6, pp. 1119-1130, 2004.

[4] Y. Liu, D. Tipper, and P. Siripongwutikorn, "Approximating optimal spare capacity allocation by successive survivable routing," IEEE/ACM Trans. Networking, vol. 13, no. 1, pp. 198-211, Feb. 2005.

[5] S. Ramasubramanian and A. Harjani, "Comparison of failure dependent protection strategies in optical networks," Photonic Network Communications, vol. 12, no. 2, pp. 195-210, 2006.

[6] D. Wang and G. Li, "Efficient distributed bandwidth management for mpls fast reroute," IEEE/ACM Trans. Networking, vol. 16, no. 2, pp. 486-495, 2008.

[7] P. Pan, G. Swallow, and A. Atlas, "Fast reroute extensions to rsvp-te for lsp tunnels," IETF RFC 4090, May 2001.

[8] J. Doucette and W. D. Grover, "Comparison of mesh protection and restoration schemes and the dependency on graph connectivity," in Proc. IEEE DRCN, Oct. 2001, pp. 121-128.

[9] Y. Liu and D. Tipper, "Spare capacity allocation for non-linear link cost and failure-dependent path restoration," in Proc. IEEE DRCN, 2001.

[10] R. R. Iraschko and W. D. Grover, "A highly efficient path-restoration protocol for management of optical network transport integrity," IEEE J. Select. Areas Commun., vol. 18, no. 5, pp. 779-794, May 2000.

[11] W. Grover, J. Doucette, M. Clouqueur, D. Leung, and D. Stamatelakis, "New options and insights for survivable transport networks," IEEE Commun. Mag., vol. 40, no. 1, pp. 34-41, Jan. 2002.

[12] R. Martin, M. Menth, and K. Canbolat, "Capacity requirements for the one-to-one backup option in mpls fast reroute," in Proc. BroadNets, San Jose,CA, Oct. 2006

[13] M. Frederick, P. Datta, and A. Somani, "Sub-graph routing: a generalized fault-tolerant strategy for link failures in wdm optical networks," Computer Networks, vol. 50, no. 2, pp. 181-199, 2006.

[14] S. Ramamurthy and B. Mukherjee, "Survivable wdm mesh networks, part ii - restoration," in Proc. IEEE ICC, 1999, pp. 2023-2030.

[15] H. Wang, E. Modiano, and M. Médard, "Partial path protection for wdm networks: End-to-end recovery using local failure information," in Proc. IEEE Symposium on Computers and Communications (ISCC), 2002, pp. 719-725.

[16] S. S. Lumetta, M. Médard, and Y.-C. Tseng, "Capacity versus robustness: A tradeoff for link restoration in mesh networks," IEEE/OSA J. Lightwave Technol., vol. 18, no. 12, pp. 1765-1775, December 2000.

[17] W. D. Grover, "The protected working capacity envelope concept: An alternate paradigm for automated service provisioning," IEEE Commun. Mag., vol. 42, no. 1, pp. 62-69, january 2004

[18] M. Médard, R. Barry, S. Finn, W. He, and S. Lumetta, "Generalized loop-back recovery in optical mesh networks," IEEE/ACM Trans. Networking, vol. 10, no. 1, p. 164, 2002.

[19] M. Kiaei, C. Assi, and B. Jaumard, "A survey on the p-cycle protection method," Communications Surveys \& Tutorials, IEEE, vol. 11, no. 3 , pp. 53-70, 2009.

[20] B. Wu, P.-H. Ho, K. Yeung, J. Tapolcai, and H. Mouftah, "CFP: Cooperative fast protection," IEEE/OSA J. Lightwave Technol., vol. 28, no. 7 , pp. $1102-1113$, apr. 2010
[21] D. A. Schupke, W. D. Grover, and M. Clouqueur, "Strategies for enhanced dual failure restorability with static or reconfigurable p-cycle networks," in Proc. IEEE ICC, Paris, France, Jun. 2004, pp. 1628-1633.

[22] S. Ramasubramanian and A. Chandak, "Dual-link failure resiliency through backup link mutual exclusion," IEEE/ACM Trans. Networking, vol. 16, no. 1, pp. 157-169, 2008

[23] B. Wu, K. Yeung, and P.-H. Ho, "Ilp formulations for p-cycle design without candidate cycle enumeration," IEEE/ACM Trans. Networking, vol. 18, no. 1, pp. 284-295, 2010.

[24] S. Sebbah and B. Jaumard, "P-cycle based dual failure recovery in WDM mesh networks," in Proc. IFIP Working Conference on Optical Network Design \& Modelling (ONDM), 2009.

[25] C. Li, R. Ramaswami, I. Center, and Y. Heights, "Automatic fault detection, isolation, and recovery in transparentall-optical networks," IEEE/OSA J. Lightwave Technol., vol. 15, no. 10, pp. 1784-1793, 1997.

[26] C. Mas, I. Tomkos, and O. Tonguz, "Failure location algorithm for transparent optical networks," IEEE J. Select. Areas Commun., vol. 23, no. 8, pp. 1508-1519, 2005.

[27] Y. Wen, V. Chan, and L. Zheng, "Efficient fault-diagnosis algorithms for all-optical WDM networks with probabilistic link failures," IEEE/OSA J. Lightwave Technol., vol. 23, pp. 3358-3371, 2005.

[28] H. Zeng and A. Vukovic, "The variant cycle-cover problem in fault detection and localization for mesh all-optical networks," Photonic Network Communications, vol. 14, no. 2, pp. 111-122, 2007.

[29] B. Wu, K. Yeung, and P.-H. Ho, "Monitoring cycle design for fast link failure localization in all-optical networks," IEEE/OSA J. Lightwave Technol., vol. 27, no. 10, pp. 1392-1401, 2009.

[30] S. Ahuja, S. Ramasubramanian, and M. Krunz, "SRLG Failure Localization in All-Optical Networks Using Monitoring Cycles and Paths," in Proc. IEEE INFOCOM, 2008, pp. 181-185.

[31] E. A. Doumith, S. A. Zahr, and M. Gagnaire, "Monitoring-tree: An innovative technique for failure localization in WDM translucent networks," in Proc. IEEE GLOBECOM, 2010, pp. 1-6.

[32] S. Stanic, S. Subramaniam, G. Sahin, H. Choi, and H. A. Choi, "Active monitoring and alarm management for fault localization in transparent all-optical networks," IEEE Trans. on Network and Service Management, vol. 7, no. 2, pp. 118-131, 2010.

[33] P. Babarczi, J. Tapolcai, and P.-H. Ho, "SRLG failure localization with monitoring trails in all-optical mesh networks," in Proc. IEEE DRCN, 2011, pp. 188-195.

[34] C. Mas and P. Thiran, "An efficient algorithm for locating soft and hard failures in wdm networks," IEEE J. Select. Areas Commun., vol. 18, no. 10, pp. 1900-1911, 2002.

[35] S. Ahuja, S. Ramasubramanian, and M. Krunz, "Single link failure detection in all-optical networks using monitoring cycles and paths," IEEE/ACM Trans. Networking, vol. 17, no. 4, pp. 1080-1093, 2009.

[36] B. Wu, P.-H. Ho, J. Tapolcai, and P. Babarczi, "Optimal allocation of monitoring trails for fast srlg failure localization in all-optical networks," in Proc. IEEE GLOBECOM, 2010.

[37] B. Wu, P.-H. Ho, K. Yeung, J. Tapolcai, and H. Mouftah, "Optical Layer Monitoring Schemes for Fast Link Failure Localization in All-Optical Networks," IEEE Communications Surveys \& Tutorials, vol. 13, no. 1, pp. $114-125$, quarter 2011.

[38] N. Harvey, M. Patrascu, Y. Wen, S. Yekhanin, and V. Chan, "NonAdaptive Fault Diagnosis for All-Optical Networks via Combinatorial Group Testing on Graphs," in Proc. IEEE INFOCOM, 2007, pp. 697705.

[39] C. Assi, Y. Ye, A. Shami, S. Dixit, and M. Ali, "A hybrid distributed fault-management protocol for combating single-fiber failures in mesh based DWDM optical networks," in Proc. IEEE GLOBECOM, 2002, pp. 2676-2680.

[40] J. Tapolcai, B. Wu, and P.-H. Ho, "On monitoring and failure localization in mesh all-optical networks," in Proc. IEEE INFOCOM, Rio de Janero, Brasil, 2009, pp. 1008-1016.

[41] C. Machuca and M. Kiese, "Optimal placement of monitoring equipment in transparent optical networks," in Proc. IEEE DRCN, 2009, pp. 1-6.

[42] J. Tapolcai, P.-H. Ho, L. Rónyai, and B. Wu, "Network-wide local unambiguous failure localization (nwl-ufl) via monitoring trails," IEEE/ACM Transactions on Networking, 2012.

[43] B. Wu, P.-H. Ho, J. Tapolcai, and X. Jiang, "A novel framework of fast and unambiguous link failure localization via monitoring trails," in IEEE INFOCOM WIP, San Diego, 2010. 
[44] D. Papadimitriou and E. Mannie, "Analysis of generalized multi-protocol label switching (gmpls)-based recovery mechanisms (including protection and restoration)," RFC 4428, March, Tech. Rep., 2006.

[45] P. Chołda, J. Tapolcai, T. Cinkler, K. Wajda, and A. Jajszczyk, "Quality of resilience QoR as a network reliability characterization tool," IEEE Network Magazine, vol. 23, no. 2, pp. 11-19, March/April 2009.

[46] B. Wu, P.-H. Ho, and K. Yeung, "Monitoring trail: On fast link failure localization in all-optical WDM mesh networks," IEEE/OSA J. Lightwave Technol., vol. 27, no. 18, pp. 4175-4185, 2009.

[47] J. Tapolcai and L. Rónyai, "Generalized non-adaptive combinatorial group testing (gncgt)," Budapest University of Technology and Economics, Tech. Rep., 2012. [Online]. Available: http://opti.tmit.bme.hu/ tapolcai/papers/tapolcai2012ncgt.pdf

[48] W. D. Grover, Mesh-based Survivable Networks: Options and Strategies for Optical, MPLS, SONET and ATM Networking. Upper Saddle River, New Jersey: Prentice Hall PTR, 2003.

[49] M. Pióro and D. Medhi, Routing, Flow, and Capacity Design in Communication and Computer Networks. The Morgan Kaufmann Series in Networking, 2004.

\section{APPENDIX}

Lemma 2: Then $f(t)=\frac{2 t}{t+1}\left(\log _{2}(t)+\frac{m-t}{t}\right)$ is a decreasing function of $t$ for $1 \leq t \leq \frac{m}{2}$.

Proof: One can verify the lemma for $m<16$. For $m \geq$ 16 we have

$f^{\prime}(t)=\frac{2}{(t+1)^{2}}\left(\log _{2} t+\frac{m-t}{t}\right)+\frac{2 t}{t+1}\left(\frac{1}{t \ln 2}-\frac{m}{t^{2}}\right)$,

and

$$
\frac{(t+1)^{2}}{2} f^{\prime}(t)=\log _{2} t+\frac{m-t}{t}+\frac{t+1}{\ln 2}-\frac{m(t+1)}{t} .
$$

Multiplying by $t$ we obtain

$$
\begin{aligned}
& \frac{t(t+1)^{2}}{2} f^{\prime}(t)=t \log _{2} t+m-t+\frac{t(t+1)}{\ln 2}-m(t+1)= \\
= & t \log _{2} t-t+\frac{t(t+1)}{\ln 2}-m t=t\left(\log _{2} t-1+\frac{(t+1)}{\ln 2}-m\right) .
\end{aligned}
$$

We have to show that

$$
\log _{2} t-1+\frac{(t+1)}{\ln 2}-m \leq 0
$$

on $\left[1, \frac{m}{2}\right]$. As the function of $t$ on the left hand side is increasing on the interval, it is enough to verify (21) for $t=m / 2$. This follows by noting that the function $h(m):=$ $\log _{2} m-2+\frac{(m / 2+1)}{\ln 2}-m$ is decreasing for $m>8$, and $h(16)<0$.

Lemma 3: Let $n, m>0$ be fixed real numbers. Then $\frac{2 t}{n+1}\left(\log _{2} t+\frac{m-t}{t}\right)$ is an increasing function of $t$ for on $[1, \infty]$.

Proof: Clearly it is enough to show that $g(t)=$ $t\left(\log _{2} t+\frac{m-t}{t}\right)$ is increasing. We have

$$
\begin{gathered}
g^{\prime}(t)=\log _{2} t+\frac{m-t}{t}+t\left(\frac{1}{t \ln 2}-\frac{m}{t^{2}}\right)= \\
=\log _{2} t+\frac{1}{\ln 2}-1>\log _{2} t+0.44>0
\end{gathered}
$$

on $[1, \infty]$. 